\title{
Kann die Spitalmedizin noch liberal sein?
}

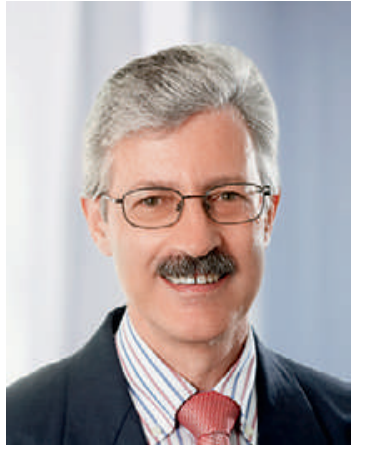

Die vierte Version der Tarifstruktur für die Akutsomatik wurde vor kurzem vom Verwaltungsrat der SwissDRG AG angenommen. Sie stellt erneut eine wichtige Entwicklung im Vergleich zu den bisherigen Versionen dar. SwissDRG 4.0 bedeutet zweifellos mehr Zusatzentgelte und wertet die ärztlichen Leistungen beträchtlich auf. Dies führt umgekehrt jedoch vor allem im universitären Bereich zu sinkenden Einnahmen bei den techniklastigen Disziplinen. Ferner wurden die Anlagennutzungskosten erstmals in die Berechnungen einbezogen, allerdings leider auf der Grundlage äusserst unterschiedlicher Daten.

Diese Entwicklung, die einige freut und andere beunruhigt, darf nicht dazu führen, dass wir das gesteckte Ziel aus den Augen verlieren: eine Pauschalvergütung, die stationäre Behandlungsfälle leistungsgerecht abbildet. So ist punkto Datenerfassung noch einiges zu tun, sowohl was die Berechnung des Wertes der Leistung betrifft, als auch was die Vollständigkeit ihrer Erfassung bei den Kodierungs- und Fakturierungsprozessen anbelangt. Wir müssen uns weiter intensiv um eine differenzierte Tarifstruktur auf Landesebene bemühen, um darauf verzichten zu können, Unschärfen durch differenzierte Baserates auszugleichen.

\section{Zwar weist die Nutzung der verfügbaren \\ Ressourcen noch Rationalisierungspotential auf, aber die Grenzen bei der Jagd nach Optimierung scheinen erreicht.}

Seit 2012 hat die neue Tarifstruktur SwissDRG alle Beteiligten auf eine harte Probe gestellt. Weil diese bei den Leistungserbringern eine umfassende Rationalisierung eingeleitet hat, müssen die Patientinnen und Patienten mit erschwerenden Umständen rechnen. Und dies, obwohl sie mehr rechtliche Freiheiten haben. Die Versicherer wiederum sehen sich mit der moralischen Verantwortung für eine eventuelle Rationierung konfrontiert, die durch ihre Unnachgiebigkeit ausgelöst werden könnte.

In diesem Spannungsfeld widersprüchlicher Forderungen stehen auch die Spitalärztinnen und -ärzte. Während ihrer gesamten Aus- und Weiterbildung werden sie darin unterwie- sen, eine einwandfreie Behandlungsqualität zu erzielen, deren Niveau langfristig durch eine intensive Fortbildung zu erhalten ist - nur wenige Berufe haben derart hohe Anforderungen. Doch die wirtschaftlichen Anforderungen im Berufsalltag treiben sie zu immer schnellerem Arbeiten bei zunehmend reduzierten personellen und materiellen Ressourcen. Zwar weist die optimale Nutzung der verfügbaren Ressourcen noch Rationalisierungspotential auf, aber die Grenzen der Jagd nach weiteren Optimierungsmöglichkeiten scheinen erreicht. Der Handlungsspielraum der Spitaldirektionen und folglich auch der leitenden Ärztinnen und Ärzte ist stark geschrumpft.

\section{Die Herausforderungen bleiben zahl- reich, insbesondere in den öffentlichen Spitälern, die immer schlechter ver- gütete Leistungen garantieren müssen.}

Auch an das Management sind die Herausforderungen gestiegen. Insbesondere im öffentlichen Bereich sind Grundleistungen zu garantieren, die immer schlechter vergütet werden. Dadurch werden oft die erforderlichen Entscheidungen verhindert. Gleichzeitig ist es nicht möglich, in allen Spitälern des Landes auf Unrentables zu verzichten. Die Tarifverhandlungen sind schwierig und enden oft in wenig zufriedenstellenden Kompromissen. Sie kommen durch die Schlichtung der Kantone zustande, werden aber alsbald wieder gerichtlich angefochten. Die unterschiedlichen Sichtweisen der Partner nähren den staatlichen Interventionismus. In erster Linie kantonal, aber auch eidgenössisch durch die subsidiäre Kompetenz.

Was ist also nun angebracht? Unabdingbar ist eine angemessene Granularität der Tarifstruktur, die das gegenseitige Vertrauen unter den Partnern, damit sie sich im System wiederfinden, stärkt. Die Spitalleitungen müssen aufgeblähte Strukturen vermeiden und sich funktionell ausrichten, wobei sie sich flexibel dem medizinisch-pflegerischen Kontext anpassen sollen. In einer allgemein angespannten finanziellen Lage liesse sich auf diese Weise ein Freiraum für die Spitalmedizin erhalten, der ein Garant für die Entfaltung der Ärztinnen und Ärzte sowie für die Qualität der Behandlung der Patientinnen und Patienten ist.

Dr. med. Pierre-François Cuénoud, Vizepräsident der FMH, Departementsverantwortlicher Tarife und Gesundheitsökonomie Spitalärzte 